\title{
Microglial Cx3cr1 knockout prevents neuron loss in a mouse model of Alzheimer's disease
}

\author{
Martin Fuhrmann ${ }^{1,4}$, Tobias Bittner ${ }^{1,4}$, Christian K E Jung ${ }^{1}$, Steffen Burgold ${ }^{1}$, Richard M \\ Page $^{2}$, Gerda Mitteregger ${ }^{1}$, Christian Haass ${ }^{2}$, Frank M LaFerla ${ }^{3}$, Hans Kretzschmar ${ }^{1}$, and \\ Jochen Herms ${ }^{1}$ \\ ${ }^{1}$ Center of Neuropathology and Prion Research, Ludwig-Maximilians-University, Munich, \\ Germany \\ ${ }^{2}$ German Center for Neurodegenerative Diseases Munich, Ludwig-Maximilians-University, \\ Munich, Germany \\ ${ }^{3}$ Department of Neurobiology and Behavior, University of California, Irvine, California, USA
}

\begin{abstract}
Microglia, the immune cells of the brain, can have a beneficial effect in Alzheimer's disease by phagocytosing amyloid- $\beta$. Two-photon in vivo imaging of neuron loss in the intact brain of living Alzheimer's disease mice revealed an involvement of microglia in neuron elimination, indicated by locally increased number and migration velocity of microglia around lost neurons. Knockout of the microglial chemokine receptor $C x 3 \mathrm{crl}$, which is critical in neuron-microglia communication, prevented neuron loss.
\end{abstract}

It has long been thought that microglia, the immune effector cells of the brain, stay in a resting state before being activated by different insults. This view has changed, as it has been shown that microglia actively survey their surrounding brain parenchyma by protracting and retracting their fine processes under healthy conditions ${ }^{1}$. In Alzheimer's disease, microglia represent a double-edged sword. On the one hand, microglia can have a beneficial effect by secreting neurotrophic factors and phagocytosing amyloid beta $(A \beta)^{2}$, the latter of which remains controversial ${ }^{3}$. On the other hand, microglia may also be neurotoxic ${ }^{4}$. Little is known about the neurotoxic role of microglia in Alzheimer's disease. Human peripheral blood monocytes that are stimulated with $A \beta$ induce neuron loss in vitro ${ }^{5}$. Neurons cultured without microglia are resistant to $A \beta$-induced neurotoxicity 6 .

(C) 2010 Nature America, Inc. All rights reserved.

Correspondence should be addressed to J.H. (jochen.herms@ @med.uni-muenchen.de).

${ }^{4}$ These authors contributed equally to the work.

Note: Supplementary information is available on the Nature Neuroscience website.

AUTHOR CONTRIBUTIONS

M.F. and T.B. conducted the experiments and wrote the manuscript. C.K.E.J. and S.B. provided technical assistance. R.M.P. performed A $\beta$ measurements. G.M., H.K., C.H. and F.M.L. provided mouse models and helpful discussion. J.H. coordinated the research and supervised the project.

COMPETING FINANCIAL INTERESTS

The authors declare no competing financial interests. 
To shed more light on the neurotoxic role of microglia in Alzheimer's disease, we simultaneously monitored the fate of individual microglia and neurons in the intact brain of a triple-transgenic Alzheimer's disease mouse model ${ }^{7}$ (3xTg-AD: $P S 1_{M 146 V}$ knockin, transgenic $A P P_{S w e}$ and $\operatorname{tau}_{P 301 L}$ ) over a period of 1 month by two-photon in vivo imaging 8 (Supplementary Fig. 1 and Supplementary Video 1). To fluorescently label neurons with yellow fluorescent protein (YFP) and microglia with green fluorescent protein (GFP), we intercrossed these mice with two additional transgenic mice to yield a quintuple-transgenic (5xTg) mouse model with a $P S 1_{M 146 V}$ knockin, gfp inserted into $C x 3 c r 1$, transgenic $A P P_{S w e}, \operatorname{tau}_{P 301 L}$ and $y f p$ (Supplementary Methods and Supplementary Fig. 2). The transgenic mice with GFP-labeled microglia also enabled us to interfere with neuronmicroglia communication, as $g f p$ was inserted into the chemokine receptor $C \times 3 c r l$ locus, leading to $\mathrm{CX}_{3} \mathrm{CR} 1$ deficiency in homozygous $5 \mathrm{xTg}-\mathrm{C} 33 \mathrm{crl}^{-/-}$mice. $\mathrm{CX}_{3} \mathrm{CR} 1$ is the unique receptor for fractalkine/CX $\mathrm{CX}_{3} \mathrm{CL} 1$, which is expressed in neurons and presumably acts as a membrane-bound adhesion molecule and/or cleaved chemoattractant and is important for recruiting $\mathrm{CX}_{3} \mathrm{CR} 1$-expressing microglia to injured neurons ${ }^{9,10}$.

We were able to detect and observe neuron loss simultaneously with surrounding microglia in living mice (Fig. 1a). A significant loss of 9.6 \pm 2.9 YFP-positive layer III neurons per $\mathrm{mm}_{3}$, representing $1.8 \%(n=626, P<0.01)$, was observed in $5 \times \mathrm{xTg}-\mathrm{Cx} 3 \mathrm{crl} 1^{* *+/-}$ mice at 4 6 months of age (Fig. 1a,b). None of the control mice, $\mathrm{Cx}_{3} \mathrm{crl}^{+/-}$or $\mathrm{Cx}_{3} \mathrm{crl}^{-/-}$, exhibited neuron loss over the 28-d imaging period (Fig. 1b). Knockout of $\mathrm{Cx} 3 \mathrm{crl}$ rescued the neuron loss in 5xTg- $\mathrm{Cx} 3 \mathrm{crl}^{-/-}$mice, indicating that microglia are necessary for neuron elimination (Fig. 1b). At this age, layer III neurons of $5 \mathrm{xTg}-\mathrm{Cx} 3 \mathrm{crl}$ mice were immunoreactive for intracellular $\mathrm{A} \beta$ but lacked hyperphosphorylated tau and extracellular amyloid plaques (Supplementary Fig. 3). Intact neurons that received the identical laser power were detected next to lost neurons, ruling out the possibility of phototoxicity (Fig. 1a). Moreover, we used postmortem Nissl and DAPI staining on previously imaged neurons and found that the elimination of the neuron was the cause of the neuronal signal loss in $100 \%$ of the analyzed cases ( $n=4$ mice) rather than just a loss of YFP expression (Supplementary Fig. 4).

To quantify the microglia density and velocity around lost neurons, we tracked individual microglia in 5xTg and control mice over a 28-d period (Fig. 1c-f and Supplementary Video 2). In $5 \times \mathrm{Tg}-\mathrm{C} 33 \mathrm{crl}^{+/-}$mice, the density of microglia significantly increased $(P<0.001)$ over time around lost neurons from $1.3 \pm 0.3$ to $3.4 \pm 0.2 \times 10^{4}$ microglia per $\mathrm{mm}^{3}$ but not in areas more than $100 \mu \mathrm{m}$ distant (Fig. $1 \mathrm{~g}$ ). In $5 \times \mathrm{Tg}-\mathrm{Cx} 3 \mathrm{crl}^{-/-}, \mathrm{Cx} 3 \mathrm{crl}^{+/-}$and $\mathrm{Cx}_{3} \mathrm{crl}^{-/-}$ mice, where layer III neuron loss was not detected, microglia density stayed at a constant level of $1.5 \pm 0.2 \times 10^{4}$ microglia per $\mathrm{mm}^{3}$ (Fig. $1 \mathrm{~g}$ ). Notably, we found that the microglia density increased significantly $(P<0.001)$ before neuron elimination (Fig. 1g). In addition, microglia migration to the lost neuron stopped after neuron elimination (Supplementary Fig. 5). Moreover, the migration velocity of microglia around lost neurons in $5 \times \mathrm{Tg}-\mathrm{Cx} 3 \mathrm{crl} 1^{+/-}$ mice was more than twofold greater $\left(1.05 \pm 0.03 \mu \mathrm{m} \mathrm{d}^{-1}\right)$ than in areas more than $100 \mu \mathrm{m}$ distant $\left(0.41 \pm 0.07 \mu \mathrm{m} \mathrm{d}^{-1}\right)$, in $C x 3 \mathrm{crl}^{+/-}$mice $\left(0.22 \pm 0.03 \mu \mathrm{m} \mathrm{d}^{-1}\right)$ and in $C x 3 \mathrm{crl}^{-/-}$ mice $\left(0.36 \pm 0.07 \mu \mathrm{m} \mathrm{d}^{-1}\right)$. This increase was rescued by $C x 3 c r 1$ knockout in $5 \mathrm{xTg}$ Cx3 $\mathrm{crl}^{-/-}$mice $\left(0.4 \pm 0.03 \mu \mathrm{m} \mathrm{d}^{-1}\right.$; Fig. 1h). 
As microglia have been shown to phagocytose $A \beta^{11}$, we tested the effect of fractalkine receptor knockout on $\mathrm{A} \beta$ levels. The levels of insoluble $\mathrm{A} \beta 40$ were increased in both $5 \mathrm{xTg}-$

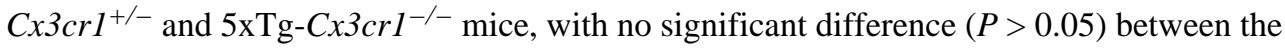
two groups (Fig. 1i). Therefore, $C x 3 c r l$ knockout appears not to interfere with the A $\beta$ phagocytosing activity of microglia.

As a measure of the microglial surveying capacity, we analyzed the total fine process turnover rate (TOR; Fig. 1j, Supplementary Videos 3 and 4 and Supplementary Methods). $\mathrm{Cx} 3 \mathrm{crl}^{+/-}$and $5 \times \mathrm{Tg}-\mathrm{Cx} 3 \mathrm{crl} \mathrm{I}^{+/-}$mice had a comparable TOR of $39 \pm 1 \%$ versus $37 \pm 1 \%$. Cx3crl knockout in either wild-type $(38 \pm 1 \%)$ or $5 x \operatorname{Tg}(34 \pm 3 \%)$ mice did not change the TOR significantly $(P>0.05$; Fig. 1k). However, there was a significant reduction $(P<$ $0.001)$ in the TOR per cell of individual microglia from $5 \times \mathrm{Tg}-C \times 3 \mathrm{crl}{ }^{+/}-$mice, which was rescued by $\mathrm{Cx} 3 \mathrm{crl}$ knockout in $5 \times \mathrm{Tg}-\mathrm{C} \times 3 \mathrm{crl} 1^{-/-}$mice (Fig. 11). This finding is probably a result of the fact that the number of microglia increased around disappearing neurons in $5 \times \mathrm{Tg}-\mathrm{Cx} 3 \mathrm{CrI}^{+/-}$mice (Fig. $1 \mathrm{~g}$ ), indicating that the screening of brain tissue is just carried out by more cells.

By simultaneously recording microglia and neurons in their intact environment using repetitive two-photon in vivo imaging, we found that microglia are involved in Alzheimer's disease-associated cortical layer III neuron loss. Analyzing the kinetics of microglia revealed that they are recruited to the neuron before and not after the elimination of the neuron and with increased migration velocity. However, whether peripheral macrophages or proliferating microglia underlie increased numbers remains an open question. Notably, our results demonstrate for the first time, to the best of our knowledge, a role for the microglial chemokine receptor $\mathrm{CX}_{3} \mathrm{CR} 1$ in Alzheimer's disease- associated neuron loss. Obviously, neuron elimination depends on an intact communication between the microglia and neuron via fractalkine and $\mathrm{CX}_{3} \mathrm{CR} 1$, as $\mathrm{CX}_{3} \mathrm{CR} 1$ deficiency prevented layer III neuron loss in 5xTg$\mathrm{Cx} 3 \mathrm{crl}^{-/-}$mice. However, the effect of wild-type $\mathrm{CX}_{3} \mathrm{CR} 1$ in $5 \times \mathrm{Tg}$ mice remains unknown. Under neuroinflammatory conditions other than Alzheimer's disease, $C x 3 c r l$ knockout has been shown to increase ${ }^{12}$ or decrease ${ }^{13}$ neurotoxicity, as well as have no effect ${ }^{14,15}$. Therefore, various neuroinflammatory conditions affect neuronmicroglia communication via fractalkine and $\mathrm{CX}_{3} \mathrm{CR} 1$ differently. $\mathrm{Cx} 3 \mathrm{crl}$ knockout did not change the elevated $\mathrm{A} \beta$ levels in 5xTg mice, suggesting that the phagocytosing activity of microglia was not altered or was not involved ${ }^{3}$. As $\mathrm{CX}_{3} \mathrm{CR} 1$ deficiency prevented neuron loss, $\mathrm{CX}_{3} \mathrm{CR} 1$ may serve as a target for the development of new therapeutic strategies for the treatment of neuron loss in Alzheimer's disease.

\section{Supplementary Material}

Refer to Web version on PubMed Central for supplementary material.

\section{Acknowledgments}

We thank S. Oddo for generously providing the 3xTg-AD mice and P. Thevnaz and E. Meijering for the development of the ImageJ plugins stackreg and MTrackJ. This work was supported by grants from the Deutsche Forschungsgemeinschaft (SFB 596, A13), the German Federal Ministry of Education and Research (Bundesministerium für Bildung und Forschung, 01GZ0713, 13N9268), the German Federal Ministry of 
Economics and Technology (Bundesministerium für Wirtschaft und Technologie, 16IN0675) and the European Union (Neuro. GSK3, FP-7-223276).

\section{References}

1. Nimmerjahn A, Kirchhoff F, Helmchen F. Science. 2005; 308:1314-1318. [PubMed: 15831717]

2. Hanisch UK, Kettenmann H. Nat. Neurosci. 2007; 10:1387-1394. [PubMed: 17965659]

3. Grathwohl SA, et al. Nat. Neurosci. 2009; 12:1361-1363. [PubMed: 19838177]

4. Wyss-Coray T. Nat. Med. 2006; 12:1005-1015. [PubMed: 16960575]

5. London JA, Biegel D, Pachter JS. Proc. Natl. Acad. Sci. USA. 1996; 93:4147-4152. [PubMed: 8633031]

6. Giulian D, et al. J. Neurosci. 1996; 16:6021-6037. [PubMed: 8815885]

7. Oddo S, et al. Neuron. 2003; 39:409-421. [PubMed: 12895417]

8. Fuhrmann M, et al. J. Neurosci. 2007; 27:6224-6233. [PubMed: 17553995]

9. Harrison JK, et al. Proc. Natl. Acad. Sci. USA. 1998; 95:10896-10901. [PubMed: 9724801]

10. Chapman GA, et al. J. Neurosci. 2000; 20:RC87. [PubMed: 10899174]

11. Bolmont T, et al. J. Neurosci. 2008; 28:4283-4292. [PubMed: 18417708]

12. Cardona AE, et al. Nat. Neurosci. 2006; 9:917-924. [PubMed: 16732273]

13. Dénes A, et al. J. Cereb. Blood Flow Metab. 2008; 28:1707-1721. [PubMed: 18575457]

14. Davalos D, et al. Nat. Neurosci. 2005; 8:752-758. [PubMed: 15895084]

15. Jung S, et al. Mol. Cell. Biol. 2000; 20:4106-4114. [PubMed: 10805752] 


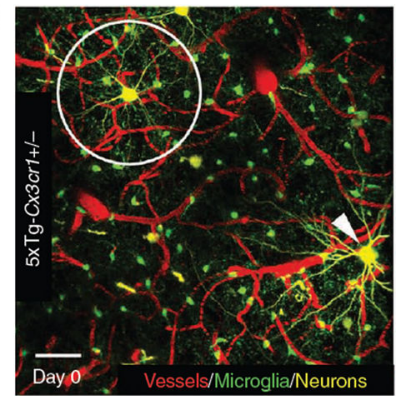

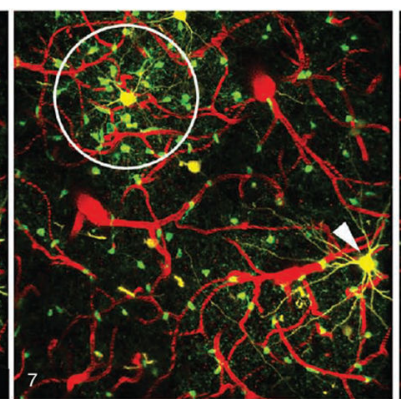
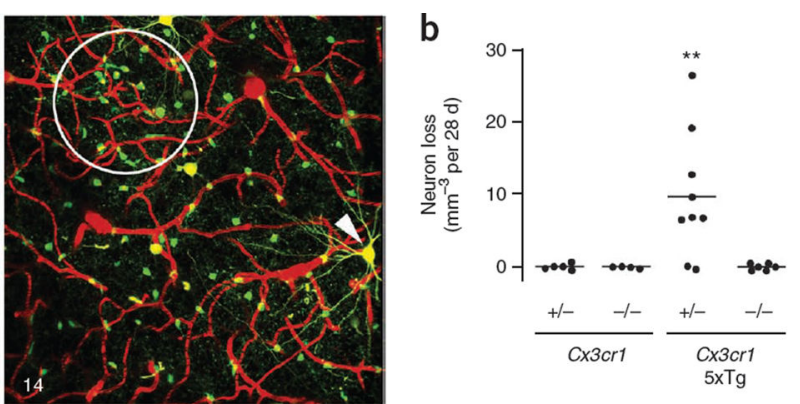

C
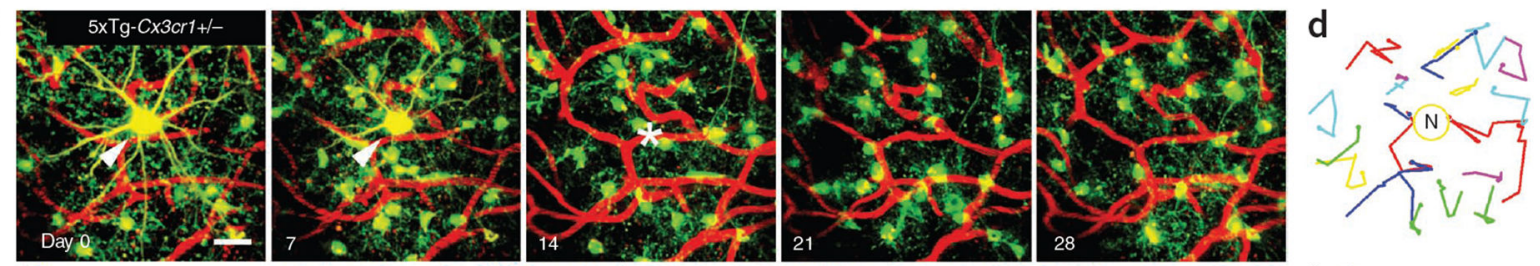

e
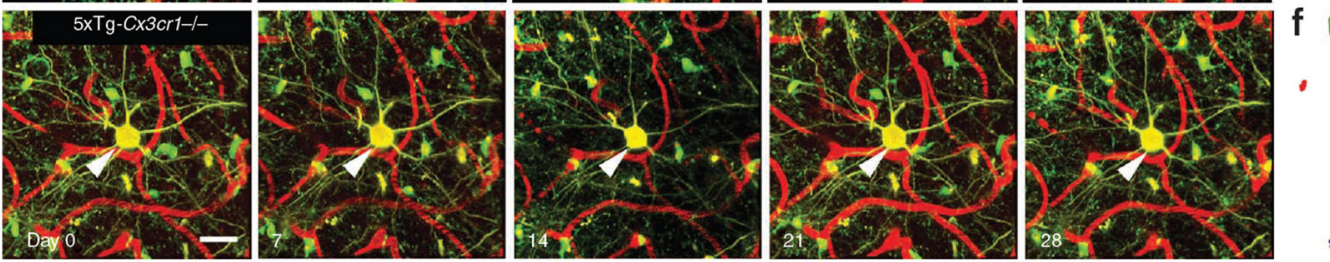

g

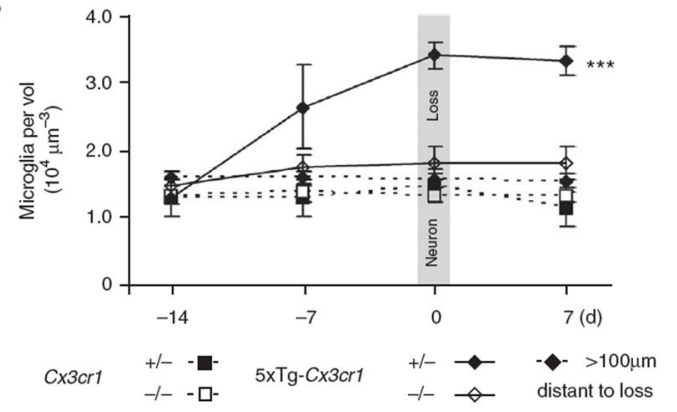

j
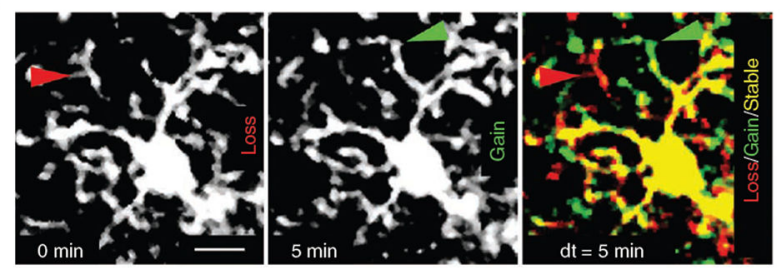

h
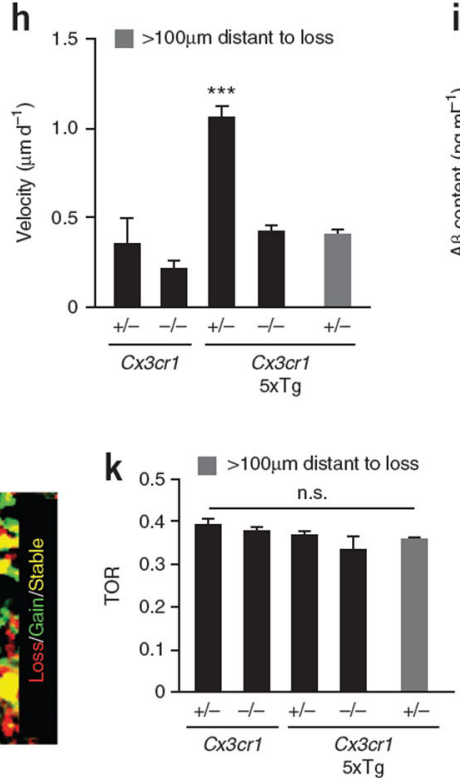

i

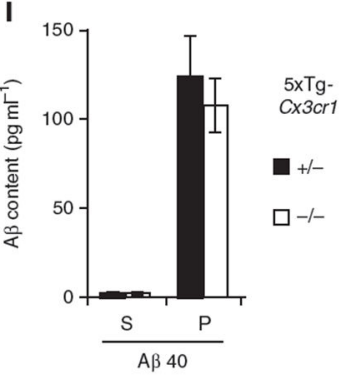

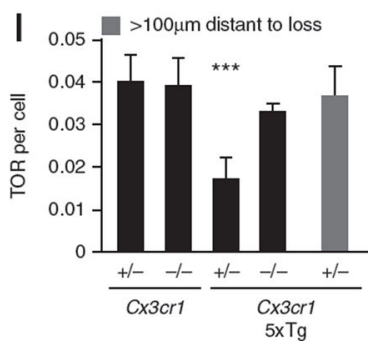

Figure 1.

Microglial $\mathrm{CX}_{3} \mathrm{CR} 1$-dependent neuron loss in 5xTg mice. (a) Fluorescence two-photon in vivo images of neurons (yellow), microglia (green) and blood vessels (red) before (day 0), at (day 7) and after (day 14) neuron loss. Circles indicate lost neurons and arrows indicate present neurons. (b) Neuron loss per volume over a 28-d imaging interval ( $n=4-9$ mice per genotype, aged 4-6 months). (c-f) High-magnification time-lapse images (c) of a lost neuron in $\mathbf{a}$ and a neuron from a $5 \times \mathrm{Tg}-\mathrm{C} x 3 \mathrm{crl}^{-/-}$mouse (e). Traces of individual microglia in $\mathrm{c}$ and $\mathbf{e}$ are shown in $\mathbf{d}$ and $\mathbf{f}$. (g) Microglia density of different genotypes ( $n=4$ per 
group) over time in a volume of $7.5 \times 10^{5} \mu \mathrm{m}^{3}$ around neurons as in $\mathbf{c}$ and $\mathbf{e}$ and more than $100 \mu \mathrm{m}$ distant. Note that microglia density increased before neuron loss. (h) Mean migration velocity of microglia somata over $28 \mathrm{~d}(n=4$ per group). (i) Soluble (S) and insoluble (P) A $\beta 40$ content in brain homogenates of $5 \times \mathrm{Tg}-\mathrm{Cx} 3 \mathrm{crl}^{+/-}$and $5 \times \mathrm{Tg}-\mathrm{Cx} 3 \mathrm{crl}^{-/-}$ mice ( $n=5$ per group). (j) Subsequent time points $(0 \mathrm{~min}, 5 \mathrm{~min})$ were superimposed $(\mathrm{dt}=5$ $\min$ ) to measure gained (green arrow), lost (red arrow) and stable (yellow area) processes. (k,l) Microglia TOR (k) and TOR per cell (l) of various genotypes ( $n=4$ mice per group) and at more than $100 \mu \mathrm{m}$ from neuron loss. Scale bars represent $20 \mu \mathrm{m}(\mathbf{a}), 10 \mu \mathrm{m}(\mathbf{c}, \mathbf{e})$ and $5 \mu \mathrm{m}(\mathbf{j}) . * * P<0.01, * * * P<0.001$. Error bars indicate s.e.m. 